\title{
EFECTOS EN EL COMERCIO DE PERECEDEROS EN LA CIUDAD DE VILLAVICENCIO GENERADOS POR LA EMERGENCIA EN LA VIA A BOGOTAAÑO 2019.
}

\section{EFFECTS ON THE TRADE OF PERISHABLE IN THE CITY OF VILLAVICENCIO GENERATED BY THE EMERGENCY ON THE ROAD TO BOGOTA YEAR 2019}

\section{Castro-Garzón, Hernando'}

Profesor, Escuela de Administración y Negocios. Facultad de Ciencias Económicas, Universidad de los Llanos, Villavicencio, Colombia.

\section{Sánchez Céspedes, Juan Manuel ${ }^{2}$}

Profesor Titular. Facultad de Ingeniería. Universidad Distrital Francisco José de Caldas. Bogotá, Colombia.

\section{Rivas Trujillo, Edwin ${ }^{3}$}

Profesor Titular. Facultad de Ingeniería. Universidad Distrital Francisco José de Caldas. Bogotá, Colombia

1 Profesor, Escuela de Administración y Negocios. Facultad de Ciencias Económicas, Universidad de los Llanos, Villavicencio, Colombia.

Correo electrónico: hcastro@unillanos.edu.co ORCID: https://orcid.org/0000-0003-1299-0489

2 Profesor Titular. Facultad de Ingeniería. Universidad Distrital Francisco José de Caldas. Bogotá, Colombia. Correo electrónico: jmsanchezc@udistrital.edu.co ORCID: https://orcid.org/0000-0001-9101-2936

3 Profesor Titular. Facultad de Ingeniería. Universidad Distrital Francisco José de Caldas. Bogotá, Colombia. Correo electrónico: erivas@udistrital.edu.co ORCID: https://orcid.org/0000-0003-2372-8056

\section{RESUMEN}

El presente estudio se desarrolló con los comerciantes presentes en la Central de Abastos de Villavicencio. Su objeto es determinar el impacto en la dimensión económica de este tipo de comercio ocasionado por la suspensión del flujo vehicular entre las ciudades de Villavicencio y Bogotá por la autopista al Llano 
acaecido en el año 2019. Así como, describir los diferentes impactos presentados durante la emergencia, las acciones que se tomaron frente a ellos, identificando los sectores del comercio afectados.

Se utilizó un enfoque cuantitativo de orden descriptivo con herramientas tipo cuestionario de preguntas para los comerciantes, este se aplicó a 285 locales que fue la muestra obtenida y una entrevista no estructurada para el director administrativo de la Central de Abastos; la información fue recolectada y posteriormente analizada a través del programa de análisis estadístico SPSS. Como conclusión se estableció que el transporte es el principal factor de incidencia en la variabilidad del precio de los perecederos, así mismo la mitad de la población intervenida enunciaron perdidas en cuanto a mercancía tanto como monetarias.

\section{PALABRAS CLAVE}

sostenibilidad, impacto, sector comercial.

\section{ABSTRACT}

The present study was developed with the merchants present at the Central de Abastos de Villavicencio. Its purpose is to determine the impact on the economic dimension of this type of trade caused by the suspension of vehicular flow between the cities of Villavicencio and Bogotá through the highway to the Llano that occurred in 2019. As well as, describe the different impacts presented during the emergency, the actions taken against them, identifying the sectors of commerce affected.

A descriptive quantitative approach was used with questionnaire-type tools for merchants, this was applied to 285 stores that was the sample obtained and an unstructured interview for the administrative director of the Central de Abastos; the information was collected and later analyzed through the statistical analysis program SPSS.
As a conclusion, it was established that transportation is the main factor of incidence in the variability of the price of perishables, likewise half of the intervened population declared losses in terms of merchandise as well as monetary losses.

\section{KEYWORDS}

sustainability, impact, commercial sector.

\section{INTRODUCCIÓN}

La vía de principal acceso al departamento del Meta a pesar de ser consideradas de cuarta generación en los últimos años ha tenido numerosos problemas, "En menos de 90 días del cierre indefinido de la vía al Llano decretado por el Gobierno Nacional, el departamento del Meta registra pérdidas de casi 2 billones de pesos" (Banguera \& Espinosa, 2019).

Villavicencio se constituye como el centro de acopio de innumerables productos y servicios, dado que conecta el centro del país con la Orinoquia, siendo la capital del departamento del Meta lo que lo convierte en una de las ciudades más importantes de la región, parta la Cámara de Comercio de Villavicencio (2018) "el Meta ha sido reconocido como la despensa agrícola de Colombia y el mayor surtidor de alimentos de Bogotá, surtiendo el $48 \%$ de la demanda" (p.3); los impactos ocasionados por los continuos bloqueos de la principal arteria terrestre, a pesar de los recursos en ella dispuestos. Según (Gutiérrez, 2015), "la inversión en la vía al Llano de los últimos años ha superado la inversión recibida antes de 1990" (p.4). Dentro de la Orinoquia, según Departamento Nacional de Planeación (2016) "el 60\% de la red vial terciaria se encuentra en tierra y de esta red $60 \%$ presenta un mal estado, el $32 \%$ en regular estado y el $8 \%$ en estado bueno" (p.3), por lo tanto se percibe que "La región Orinoquia ha mostrado tener potencial en diversos sectores agrícolas. Sin embargo, muchas veces las iniciativas se ven 
afectadas por no tener carreteras que permitan el buen transporte de sus productos" (García, 2019)

Por otro lado, los centros de acopio de perecederos son "escenarios sociales donde suceden relaciones de intercambio e interacción entre compradores y vendedores, en los cuales existen lógicas de mercadeo y negociación en relación con la circulación de mercancías y la dinámica de precios y valores" (Prieto \& Chinchilla, 2018, pág. 10). Estas interacciones se ven afectadas por la vulnerabilidad de los comercios, "Los permanentes cierres del corredor Bogotá-Villavicencio perjudican cada vez más a los comerciantes y campesinos del departamento, especialmente a los agricultores del Ariari" (Castañeda, Charrupí, \& Jiménez, 2019). Tambien se evidencia que en la central de Abastos tiene ausencia de una"visión más real de su situación y considerar alternativas de solución para minimizar las barreras de su desarrollo y crecimiento" (Romero, 2017).

El costo económico de cerrar un flujo de movilidad en una zona estratégica terrestre es alto Un día de inactividad en la vía representa pérdidas por 53 mil millones de pesos (Rodríguez, 2019) y según Barbosa(2019) "el gremio turístico pierde 1.300 millones de pesos diarios y ha tenido que despedir más del personal de los hoteles y restaurantes en la ciudad".

La vía Villavicencio- Bogotá es una de las vías más importantes para el país y la más importante para la Orinoquia Colombia; cuando "la carretera está cerrada o solo parcialmente abierta, la conectividad de esta importante región del país es reducida. Solo hay otros dos caminos que sirven como alternativa para conectar la capital del Meta con Bogotá" (Duque 2019) que "a pesar de considerarse la despensa agrícola de
Colombia, el último cierre ha mandado al infierno a muchos sectores productivos, incluyendo a la ganadería, avicultura y porcicultura, por mencionar algunas ramas pecuarias y agrarias" (Aguilar, 2019).

Este trabajo analizó el impacto que ocasionó el cierre de la vía al llano entre julio y octubre del 2019 estudiando el nivel de ingreso de los comerciantes antes y después del cierre para establecer una relación de impacto a través de la interpretación de la información recopilada previo a la situación que vivió la Central de Abastos de Villavicencio.

\section{MATERIALES Y MÉTODOS}

El método aplicado, en este manuscrito es del orden descriptivo, dado que analiza elementos particulares al todo como universo de elementos transversales, desde la observación real del fenómeno, y la utilización complementada con el análisis del mismo. Según el alcance y análisis de los resultados, se considera como descriptivo, dado que, se describen de manera significativa algunas características del fenómeno homogéneo. Además, se aplicó la técnica de investigación, estudio de caso, el cual se caracteriza en un análisis sistemático de un proceso o asunto científico de interés, en condiciones de un fenómeno homogéneo o situaciones que merecen un interés de aplicación, concreta y precisa, según la temática y el objeto de estudio a analizar.

\section{ÁREA DE ESTUDIO}

La Central de Abastos de Villavicencio establecida como el centro de acopio principal del departamento se intervino una muestra de 285 locales comerciales, esta muestra se definió con la siguiente fórmula . 


\begin{tabular}{|l|c|c|r|}
\hline \multicolumn{4}{|c|}{ DATOS } \\
\hline POBLACIÓN & $\mathrm{N}=$ & 1090 & \\
\hline NIVEL CONFIANZA & $\mathrm{z}=$ & 1,95 & $\mathbf{9 5 \%}$ \\
\hline Proporción de aceptación & $\mathrm{p}=$ & 0,5 & \\
\hline Proporción de rechazo & $\mathrm{q}=$ & 0,5 & \\
Error Máximo Admisible & $\mathrm{E}=$ & 0,05 &
\end{tabular}

\section{ESTRATEGIA METODOLÓGICA}

Se desarrolló con un enfoque cuantitativo y tuvo un diseño de investigación de tipo descriptivo, ya que se buscó describir los problemas que se presentaron durante el cierre de la vía, identificar cuales fueron los sectores más afectados, la fuente primaria provino de los propios comerciantes y las secundarias de artículos y publicaciones

\section{PROCESAMIENTO DE LA INFORMACIÓN}

La información que se obtuvo de los comerciantes a través del instrumento propuesto, fue recopilada a través de tablas y se analizó en el programa de análisis estadístico SPSS a través de gráficos y correlaciones entre las variables.

\section{RESULTADOS}

Los análisis de resultados se realizaron a partir de la tabulación realizada en Excel y de las tablas de correlación arrojadas por el programa de análisis estadístico SPSS.

Análisis Excel:

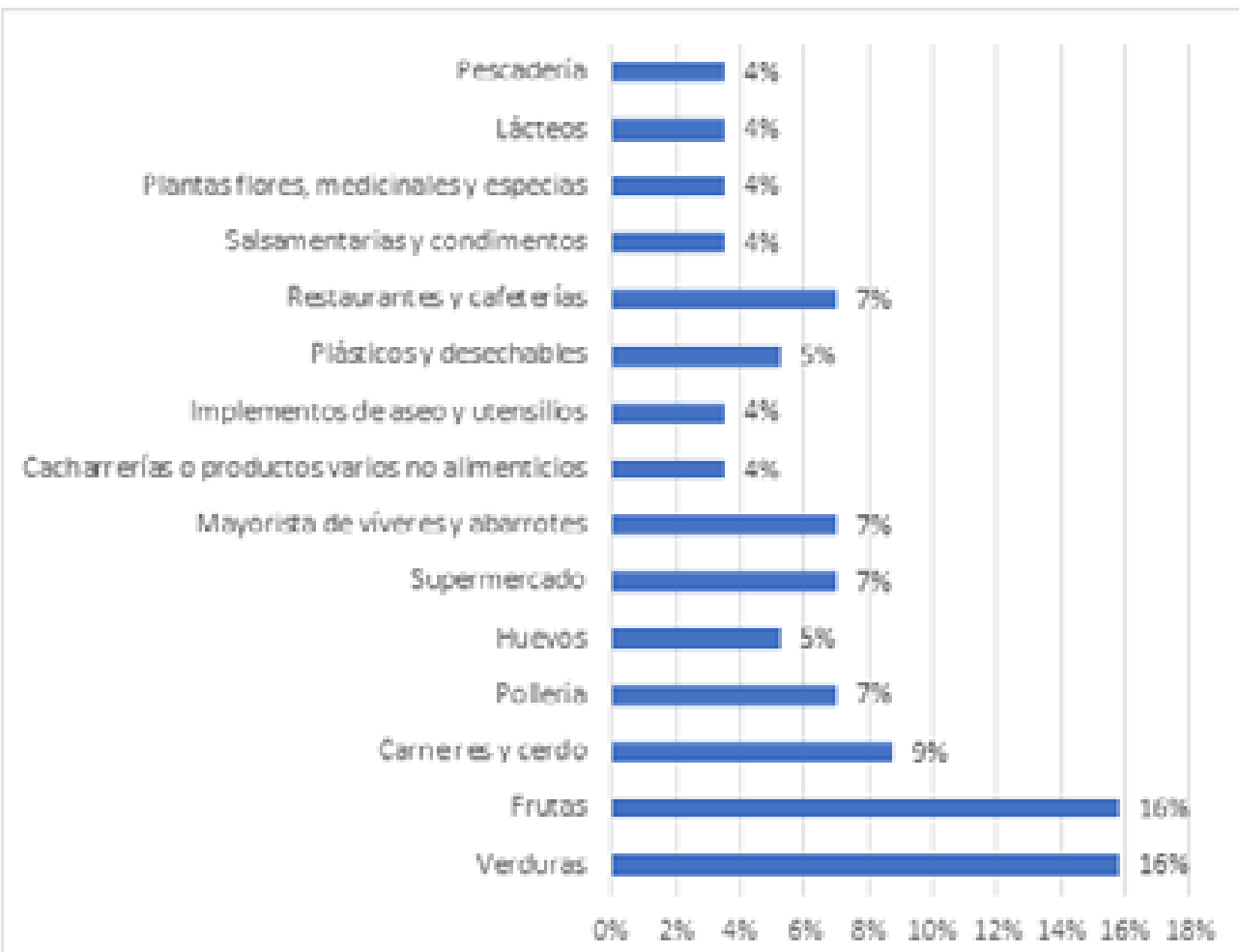

Figura 1 Tipo de negocio comercial

Fuente: los autores a partir de datos establecidos, 2020. 
Estableciendo el tamaño, así como los sectores y el flujo de productos en la Central de Abastos de Villavicencio, en donde predominó las frutas y verduras en contraste con negocios como el de la venta de huevos o puestos de flores y especias; estableciendo que los comercios más importantes son los distribuidores de papa y plátano.

Figura 2 Ingresos mensuales antes del cierre vial

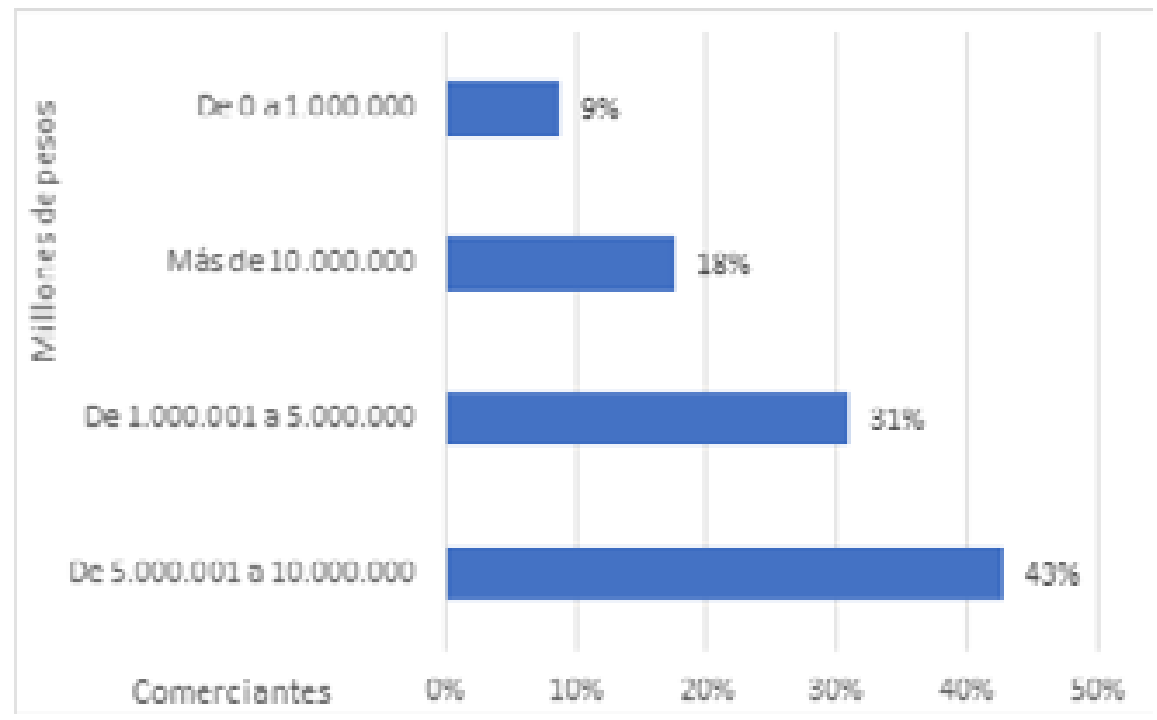

Fuente: los autores a partir de datos establecidos 2020.

Se estableció que los ingresos percibidos antes del cierre de la vía oscilaban entre más de 5 a 10 millones de pesos COP mensuales en el $43 \%$ del total de comerciantes encuestados, En relación con, (Romero, 2017) donde de acuerdo con su estudio el $47 \%$ de los comerciantes tenían ingresos en un rango de más de 300 mil hasta 500 mil pesos COP diarios, un promedio de 12 millones de pesos al mes.

Figura 3 ¿Durante el cierre total de la vía comprendido entre los meses de Julio a octubre presentó perdidas en su negocio?

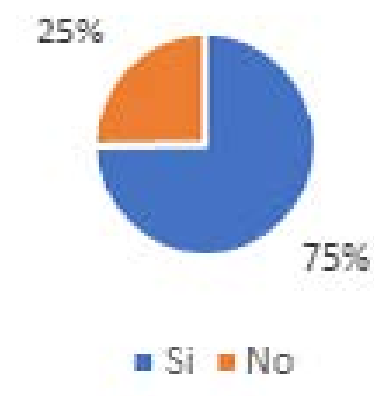

Fuente: los autores a partir de datos establecidos 2020. 
Frente a la situación del cierre total de la vía Bogotá - Villavicencio, el $75 \%$ de los encuestados presentó perdidas, el $25 \%$ resaltó que dependían muy poco del cierre de la vía.
Otro caso por los que se pueden presentar pérdidas en los negocios es cuando existen productos de movimiento lento, es decir, de baja rotación (Batista, 2016).

Figura 4 En qué porcentaje tuvo pérdidas durante el cierre vial

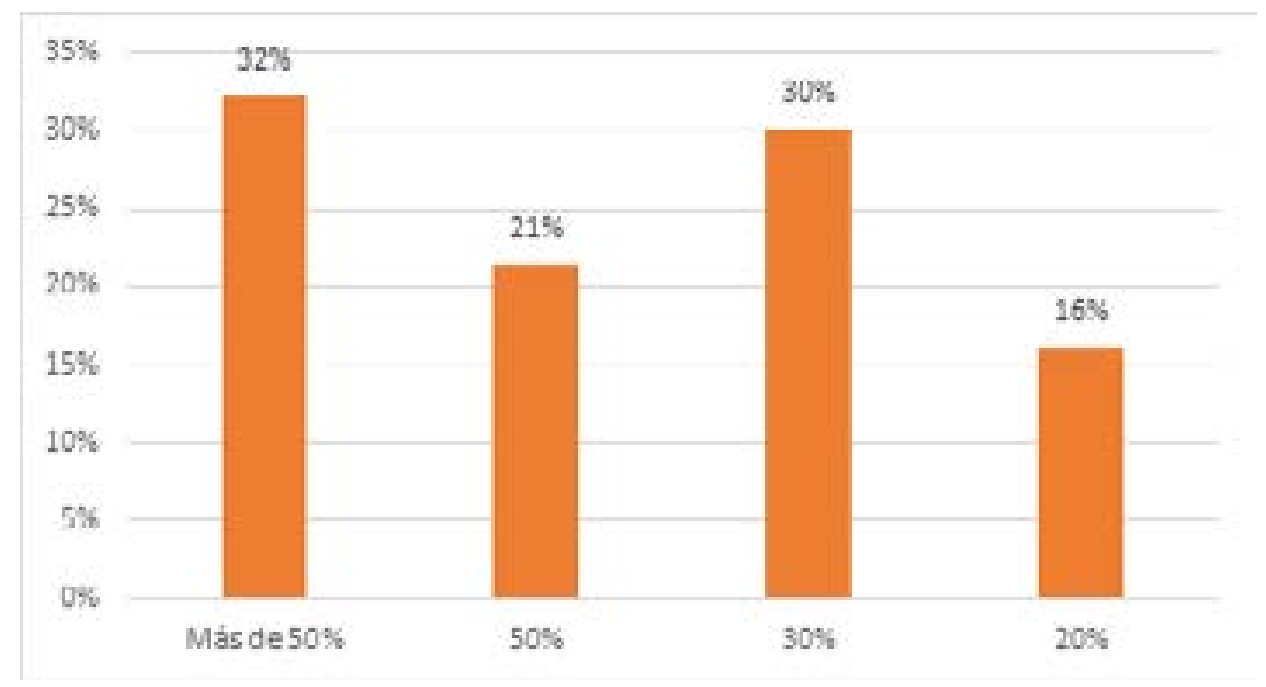

Fuente: los autores a partir de datos establecidos 2020.

Las perdidas establecidas dependieron del tipo de comercio que realizaban, así como el lugar de procedencia de los mismos. Quienes generaron pérdidas de más del $50 \%$ su abastecimiento es realizado de la zona centro del país, específicamente de Boyacá, que es el segundo departamento con más producción de papa, la disponibilidad hídrica y las bajas temperaturas son lo que convierte a Boyacá en un productor papero (Fedepapa, 2018).

Figura 5 ¿De las siguientes opciones cual afecto más su negocio?

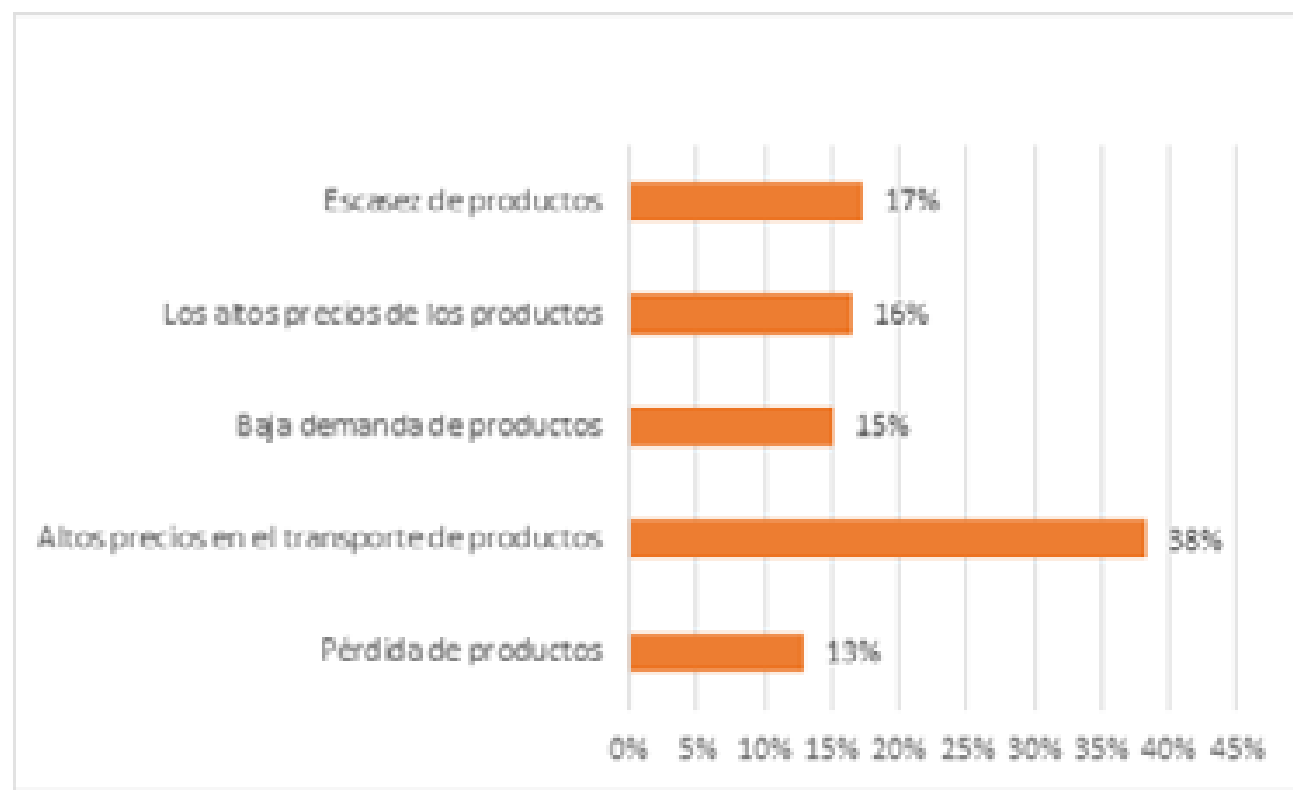

Fuente: los autores a partir de datos establecidos 2020. 
Se observo que el factor que más impacto negativo ocasiono fue los precios del transporte con un $38 \%$, adicionando con la escasez con un $17 \%$. (Guadalupe \& De la Torre Moreno, 2006) señalaron que alrededor del $50 \%$ de la producción nacional de pescados y mariscos, y $33 \%$ de las frutas y hortalizas se pierden en el país (México) por la escasez de transporte adecuado y por el número insuficiente de almacenes especializados.

Figura 6 Medidas que tomaron los comerciantes frente a la crisis

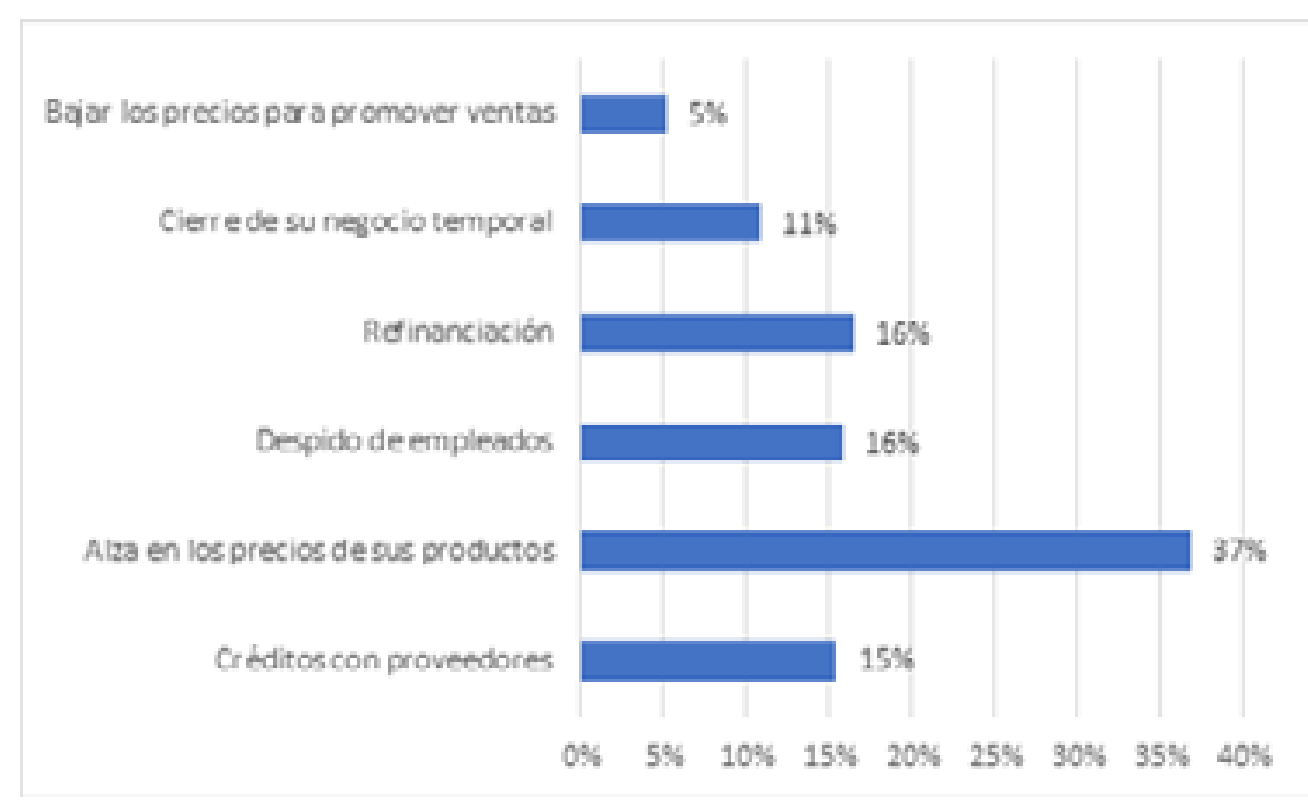

Fuente: los autores a partir de datos establecidos 2020.

Como opción para mitigar la situación la salida principal fue la subida de precios con una participación del $37 \%$ de los comerciantes encuestados, un $16 \%$ afirmó el uso de refinanciación con otros ingresos. El gobierno Nacional determinó 8 medidas para mitigar el impacto que tuvo el cierre vial, dentro de ellas, una línea adicional de Bancoldex para los departamentos de Cundinamarca, Meta y Casanare, Vichada, Arauca y Guaviare, de $\$ 200.000$ millones. Esta tendrá un plazo de cinco años, un año de gracia y tasa DTF con el fin de impulsar el capital de trabajo y la sustitución de las deudas, y también el Banco Agrario anunció prorrogas hasta de 6 meses (Bohórquez, 2019).
Figura 7 ¿Porque algunos comerciantes no presentaron pérdidas durante el cierre vial?

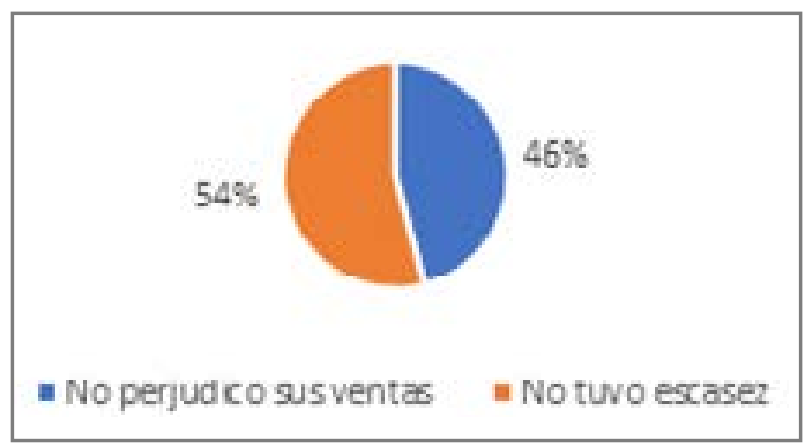

Fuente: los autores a partir de datos establecidos 2020.

Se presento un $25 \%$ de comerciantes que estableció no tener perdidas de estos el $46 \%$ ya que tienen diferentes tipos de negocios y el $54 \%$ no dependía de los productos foráneos ya que sus proveedores son de la región. Los comerciantes lograron conseguir sin aumento 
de precio, se debe a que el departamento del Meta es conocido por su producción de ganado, arroz, plátano, frutales, yuca y petróleo, además de los alevinos. (El periodico del Meta, 2019)

Figura 8 ¿Cuál fue la razón por la que su negocio no presentó pérdidas durante el cierre de la vía?

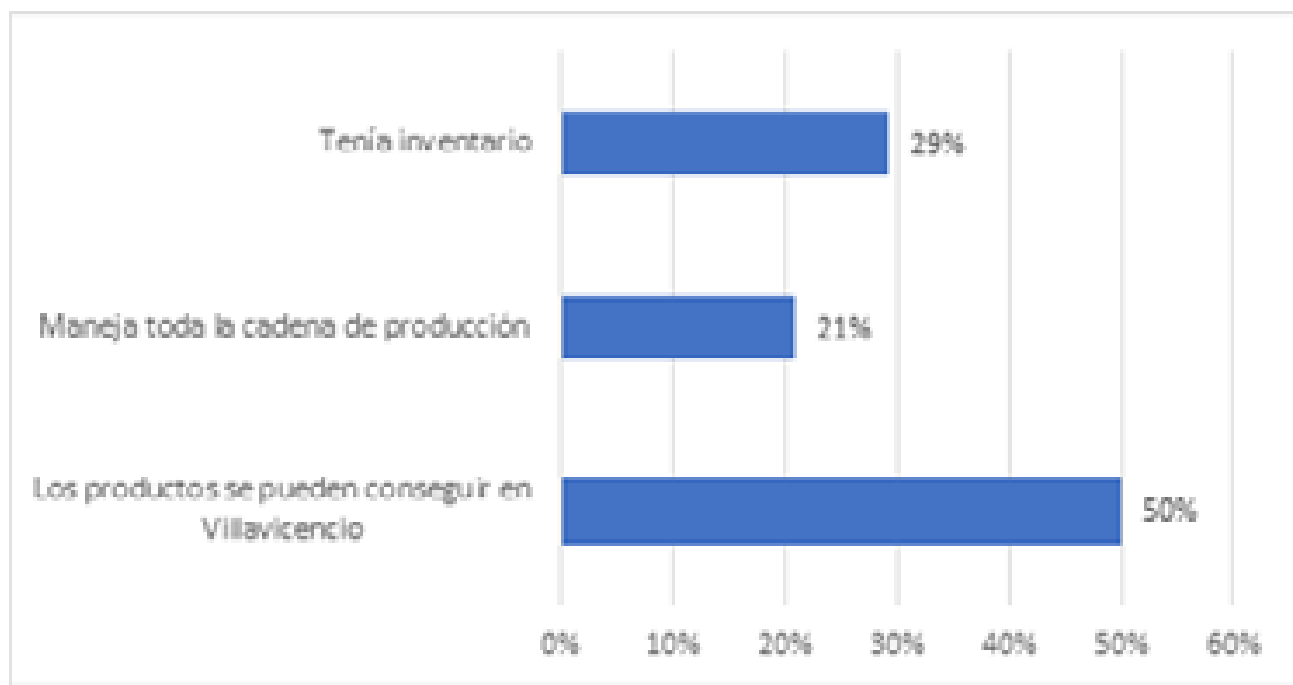

Fuente: los autores a partir de datos establecidos 2020.

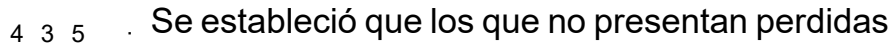
es por sus proveedores, así como que los productos no son perecederos; se evidencia que el $50 \%$ son de este tipo. En cuanto al $21 \%$ que manejan sus propias cadenas de producción pertenecían en su mayoría al sector de los cárnicos.
Figura 9 ¿Los comerciantes se han logrado recuperar de las pérdidas?

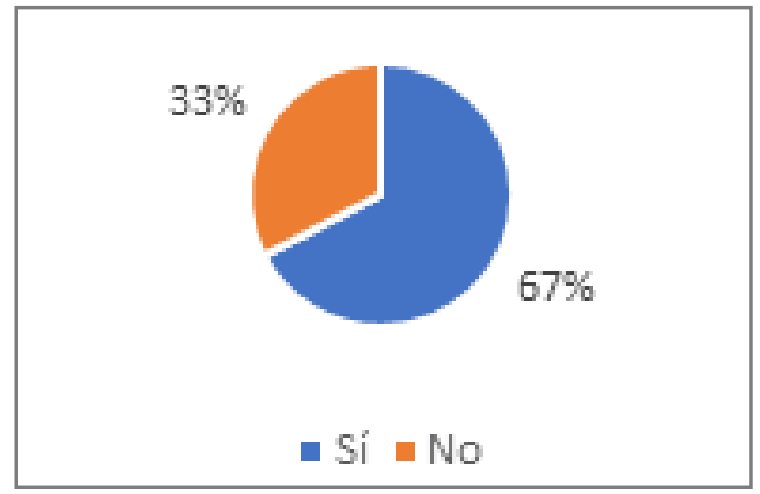

Fuente: los autores a partir de datos establecidos 2020.

La precisión frente a la recuperación es debida a la estabilidad y trayectoria de los comercios, siendo el $67 \%$ han logrado recuperarse. El $33 \%$ presentaron dificultades para estabilizarse después de la crisis. 
Figura 10 Porcentaje de recuperación de las pérdidas

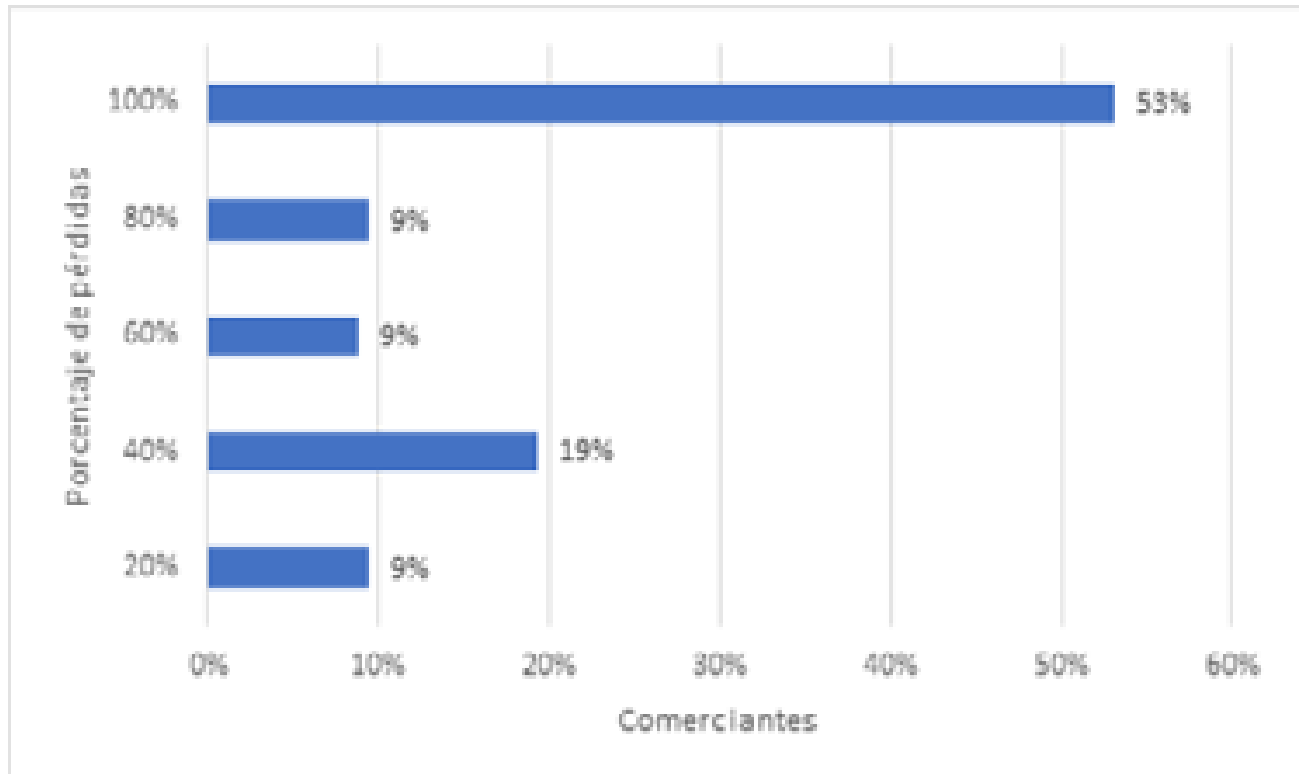

Fuente: los autores a partir de datos establecidos 2020.

La recuperación se presentó en gran medida en los comercios con tradición en el mercado, recuperados en su totalidad el $53 \%$, el $19 \%$, de Empresarios de Colombia, ANDI, solicita que se tomen las medidas necesarias y soluciones definitivas con el concesionario y así evitar los continuos cierres en la vía por causa de los derrumbes (ANDI, 2019). al tipo de productos que maneja, recurrió a préstamos. Por otro lado, la Asociación Nacional

Análisis de correlaciones: Correlación 1

Correlaciones

\begin{tabular}{|c|c|c|c|}
\hline & & $\begin{array}{l}\text { ¿Antes del } \\
\text { cierre de la } \\
\text { via estime el } \\
\text { rango de sus } \\
\text { ingresos } \\
\text { mensuales? }\end{array}$ & $\begin{array}{c}\text { ¿Si su } \\
\text { respuesta } \\
\text { anterior fue } \\
\text { si, indique } \\
\text { que } \\
\text { porcentaje } \\
\text { representa } \\
\text { sus } \\
\text { perdidas? }\end{array}$ \\
\hline $\begin{array}{l}\text { ¿Antes del cierre de la } \\
\text { via estime el rango de } \\
\text { sus ingresos } \\
\text { mensuales? }\end{array}$ & $\begin{array}{l}\text { Correlación de Pearson } \\
\text { Sig. (bilateral) } \\
\mathrm{N}\end{array}$ & $\begin{array}{r}1 \\
285\end{array}$ & $\begin{array}{r}.895^{\prime \prime} \\
.000 \\
285\end{array}$ \\
\hline $\begin{array}{l}\text { ¿Si su respuesta } \\
\text { anterior fue si, indique } \\
\text { que porcentaje } \\
\text { representa sus } \\
\text { pérdidas? }\end{array}$ & $\begin{array}{l}\text { Correlación de Pearson } \\
\text { Sig. (bilateral) } \\
\text { N }\end{array}$ & $\begin{array}{l}.895^{\circ} \\
.000 \\
285\end{array}$ & 285 \\
\hline
\end{tabular}

Fuente: los autores a partir de datos establecidos en SPSS 2020. 
Se determino una correlación directa entre la baja de ingresos y el efecto del cierre de la vía; determinando que se impactó en un cincuenta por ciento los comerciantes de la Central de Abastos fueron producto de la emergencia económica.

Correlación 2

Correlaciones

\begin{tabular}{|c|c|c|c|}
\hline & & $\begin{array}{l}\text { ¿Antes del } \\
\text { cienry de la } \\
\text { via estime ol } \\
\text { rango de sus } \\
\text { ingresos } \\
\text { mensuales? }\end{array}$ & $\begin{array}{l}\text { ¿Dy las } \\
\text { siguiontes } \\
\text { opciones cual } \\
\text { afecto más } \\
\text { su negocio? }\end{array}$ \\
\hline $\begin{array}{l}\text { ¿Antes del cierre de la } \\
\text { via estime al rango de } \\
\text { sus ingresos } \\
\text { mensuales? }\end{array}$ & $\begin{array}{l}\text { Conrelación de Pearson } \\
\text { Sig. (oilateral) } \\
\text { N }\end{array}$ & 1 & $\begin{array}{r}.902^{\prime \prime} \\
.000 \\
285\end{array}$ \\
\hline $\begin{array}{l}\text { ¿De las siguiontes } \\
\text { opciones cual afecto mas } \\
\text { su negocio? }\end{array}$ & $\begin{array}{l}\text { Correlación de Pearson } \\
\text { Sig. (bilateral) } \\
\mathrm{N}\end{array}$ & $\begin{array}{r}.902^{2 \pi} \\
.000 \\
285\end{array}$ & 1 \\
\hline
\end{tabular}

Fuente: los autores a partir de datos establecidos en SPSS 2020.

De nuevo se establece la alta relación de la incidencia del cierre frente a los ingresos estableciendo en primer lugar los altos precios del transporte lo cual está directamente relacionado con el cierre vial, seguido de la escasez de los productos.

\section{CONCLUSIONES}

El transporte de mercancías terrestre por vías alternas establece elevados costos al momento de movilizar los productos, dado que la infraestructura de estas es inadecuada, motivo por el cual se deberían generar estrategias como alianzas para el mejoramiento de las carreteras.

Tanto para los comerciantes, productores como transportadores, la importancia de los corredores viales dado que estos permitirían la generación de nuevos negocios, así como la internacionalización del departamento del Meta y la Orinoquia.
La operación de las vías, ya sean concesionadas o no, deben garantizar su funcionamiento en óptimos niveles de calidad como eficiencia; esto asegura y potencia el crecimiento económico de la población, permitiendo la integración de ser posible multimodal, ofreciendo interacción entre los centros de acopio y las regiones productoras con una adecuada presencia estatal que garantice su funcionamiento.

Para la creación de valor además de la seguridad económica de una región, en el entendido del desarrollo social, es menester la creación de vías de penetración adecuadas a su necesidades y oferta de bienes que permita la secuencia en las cadenas productivas que garanticé el adecuado comercio interregional.

El crecimiento económico va de la mano de la intervención financiera en estructura vial, no solo se habla de región sino a nivel micro, la oferta y demanda del mercado se estimula dado que la reducción de costos por un adecuado corredor 
hace que los flujos de insumos, materias primas, productos terminados tenga como beneficiario el consumidor final que recibirá bienes y servicios con precios que determinan una mejora en la calidad de vida.

AGRADECIMIENTOS: Los autores de manera especial, reconocen de manera especial a Carlos Fernando Gonzales Cuentas quien realizó el trabajo de campo y contacto con la comunidad intervenida y a la Universidad de los Llanos (Villavicencio, Colombia), por el apoyo especial en el desarrollo de la investigación.

\section{REFERENCIAS BIBLIOGRÁFICAS}

Aguilar, P. (2019). Pongámonos serios! La vía al Ilano no aguanta más cierres. Las 2 orillas. Obtenido de https://www. las2orillas.co/pongamonos-serios-lavia-al-Ilano-no-aguanta-mas-cierres/

Alcaldia de Villavicencio. (2019). Información del municipio. Villavicencio, Meta, Colombia. Recuperado el 04 de Abril de 2019, de http://www.villavicencio.gov. co/MiMunicipio/Paginas/Informaciondel-Municipio.aspx

ANDI. (2019). Comunicado de la ANDI sobre cierres continuos en la vía BogotáVillavicencio. Obtenido de http://www. andi.com.co/Home/Noticia/11416comunicado-de-la-andi-sobre-cierrescon

Banguera, J., \& Espinosa, M. (11 de 09 de 2019). El tiempo. Obtenido de https://www. eltiempo.com/colombia/otras-ciudades/ via-al-Ilano-y-su-posible-reapertura-enel-mes-de-septiembre-411006

Barbosa, W. (09 de 08 de 2019). Las pérdidas económicas en la vía al llano no paran de crecer. (R. Semana, Entrevistador) Obtenido de https://www.semana.com/ economia/articulo/millonarias-perdidas-
economicas-en-la-via-al-Ilano/630853/

Batista, Y. B. (2016). Análisis de las pérdidas en ventas generadas por productos de lento movimiento en Complejo CIMEX Moa. Cofin Habana, 10(2). Obtenido de http:// scielo.sld.cu/scielo.php?script=sci_artte xt\&pid=\$2073-60612016000200006

Bohórquez, G. K. (2019). Las ocho medidas económicas para mitigar el impacto del cierre de la vía al Llano. La republica. Obtenido de https://www.larepublica. co/economia/las-ocho-medidaseconomicas-para-mitigar-el-impactodel-cierre-de-la-via-al-llano-2874316

Cámara de cormercio de Villavicencio. (2018). Imapcto del cierre de la vía Bogotá. Villavicencio. Obtenido de http:// www.ccv.org.co/site/fileadmin/user_ upload/Investigaciones_Economicas/ IMPACTO_DEL_CIERRE_DE_ LA_VIA_BOGOTA_-_ESTUDIOS_ ECONOMICOS_-_AJUSTES_DIR_-. pdf

Castañeda, P., Charrupí, L. F., \& Jiménez, I. (2019). La Vía al Llano: el incurable dolor de cabeza del Meta. Semana rural. Obtenido de https://semanarural. com/web/articulo/via-al-llano-cierre-dela-carretera-bogota-villavicencio/641

Departamento Nacional de Planeación. (2016). Plan Maestro de la Orinoquia. Villavicencio. Obtenido de http://cccasanare.co/wp-content/ uploads/2017/02/Plan-Maestro-de-laOrinoqu\%C3\%ADa-2016.pdf

Duque Escobar, G. (2019). Qué hacer con la vía al Ilano. Obtenido de https://repositorio. unal.edu.co/handle/unal/77050 
El periodico del Meta. (2019). Meta, despensa agrícola y pecuaria. Meta, despensa agrícola y pecuaria. Obtenido de https:// periodicodelmeta.com/meta-despensaagricola-y-pecuaria/\#: :text=Tanto $\% 20$ en $\% 20$ la $\% 2$ regi \% C 3\%B 3n \% 20 como,se $\% 20$ cultivan $\% 20$ en $\% 20$ el\%20 Meta.

Fedepapa. (2018). Boyacá. BOLETÍNMENSUAL REGIONAL, 2(2). Obtenido de https:// www.fedepapa.com/wp-content/ uploads/2017/01/BOLETI\%cc\%81NRE GIONALBOYACA\%cc\%81-18.pdf

García, J. N. (2019). Las vías del llano una oportunidad para el progreso. Obtenido de https://repositorio.uniandes.edu.co/ handle/1992/31462

Guadalupe, M. P., \& De la Torre Moreno, M. E. (2006). Caracteristicas del transporte refigerado en México. Obtenido

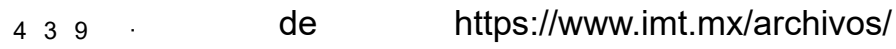
Publicaciones/PublicacionTecnica/ pt297.pdf

Gutiérrez, O. A. (2015). LA CARRETERA BOGOTÁ-VILLAVICENCIO, SU IMPACTOSOBREELORDENAMIENTO TERRITORIAL Y EL ECOSISTEMA. Luna Azul, 16. Obtenido de http://www. scielo.org.co/pdf/luaz/n40/n40a18.pdf

Navarro, L. (2019). Encuentro Gremial en Villavicencio contó con asistencia de Gobernadora del Meta y registró alta participación de palmicultores. Boletín El Palmicultor. Obtenido de https:// publicaciones.fedepalma.org/index.php/ palmicultor/article/view/12852/12736
Prieto, D. M., \& Chinchilla, M. M. (2018). El comportamiento del consumidor de las plazas de mercado de Villvicencio, Meta. Lecturas de Economía Social, 19. Obtenido de https://dialnet.unirioja.es/ servlet/articulo?codigo $=6920528$

Rodríguez, J. C. (04 de 07 de 2019). Vía al Llano: camioneros pierden $\$ 50$ mil millones. (E. n. siglo, Entrevistador) Obtenido de https://www.elnuevosiglo.com. co/articulos/07-2019-cierre-al-Ilanopierden-50-mil-millones-camioneros

Romero, D. (2017). Impacto económico y social que limita el crecimiento y desarrollo de la Central de Abastos de. Villavicencio. Obtenido de https://repositorio. unillanos.edu.co/bitstream/001/1263/1/ RUNILLANOS\%20ECO\%200451\%20 IMPACTO\%20ECONOMICO $\% 20 Y \% 20$ SOCIAL \% 20QUE \% 20LIMITA \% 20 EL $\% 20$ CRECIMIENTO $\% 20 Y \% 20$ DESARROLLO $\% 20$ DE $\% 20$ LA $\% 20$ CENTRAL\%20DE\%20ABASTOS\%20DE\%20VILLAVICENCIO.pdf 